record is of projected experiments.

People seeking an introduction to the techniques of remote geochemical exploration may find this book useful. They will not find that it has the polish of a good textbook. Perhaps that is too much to expect of an up-to-date review. GRENVILle TURNER

\section{Search for Spectra}

The Spectra and Structure of Simple Free Radicals. By Gerhard Herzberg. Pp. xi +226. (Cornell University: Ithaca and London, June 1971.) £5.25.

The 1968 Baker lectures at Cornell University by Gerhard Herzberg, now published in this slim volume, fulfil the ambition of the author to write an introduction to molecular spectroscopy. Few would argue that he is the greatest exponent of the subject still in active research, and on the basis of his three comprehensive books one might suppose that anything he does not know about molecular spectroscopy is not worth knowing.

The book has a title and a sub-title: an introduction to spectroscopy. The latter gives a better representation of its character because free radicals are used only to illustrate general features of molecular spectroscopy and little emphasis is placed on any unique features. In fact the definition of radical which has been adopted ("any species with a short life in the gas phase under normal laboratory conditions") would not be generally accepted in other branches of chemistry. For example, the molecule $\mathrm{C}_{2}$ is included but not NO. The omission of detailed discussion of electron spin resonance makes the title slightly inappropriate.

The introductory chapter covers some of the special problems that arise in the measurement of the spectra of transient species, although the book as a whole has a theoretical rather than an experimental approach to the subject. There is an enjoyable account of the search for the spectrum of $\mathrm{CH}_{2}$ which was stimulated by the observation in 1941 of some unidentified lines in the spectrum of a comet. These were finally assigned to molecule $\mathrm{C}_{3}$ but $\mathrm{CH}_{2}$ was eventually tracked down in 1961 in the flash photolysis of diazomethane.

The main part of the book is divided into three sections: diatomics, linear polyatomics and non-linear polyatomics. As an indication of the level of the book, the diatomic chapter gives an account of rotation-electronic coupling, the symmetry properties of rotational levels, of Hund's coupling cases $a$ and $b$ (but not the less important cases) and the rotational structure of electronic bands. It can therefore be seen that the book goes much further than is usual in undergraduate spectroscopy texts.
The chapters on polyatomic radicals give very good accounts of vibronic interactions (the Renner and JahnTeller effects) and of vibration-rotation interactions ( $\Lambda$ doubling and Coriolis interaction). There is also an extensive discussion of molecular orbital correlation diagrams and their relevance to the understanding of state symmetries and molecular shapes.

Finally the book is completed by a short chapter on radiationless processes: dissociation, predissociation and recombination. A description of the mechanism of these processes is given and a discussion of selection rules, but no reference has been made to the recent work on predissociation line widths which has been undertaken in several different laboratories.

The book has been elegantly produced and is a pleasure to read. It is a pity that it is so highly priced, as it should be widely used as a textbook for graduate courses in spectroscopy.

\section{J. N. MURRELL}

\section{Chemical Mutagenesis}

Chemical Mutagenesis in Mammals and Man. Edited by F. Vogel and G. Röhrborn. Pp. xiv +519. (SpringerVerlag: Berlin and New York, 1970.) 124 DM ; \$34.10.

VARIOUS expert committees have recently emphasized the critical need for mutagenicity testing of chemicals before their registration for commercial or other uses. These committees include the Task Force on Research Planning in Environmental Health Science, March, 1970; the Food and Drug Administration Advisory Committee for Protocols on Safety Evaluation, Panel on Reproduction Studies in the Safety Evaluation of Food Additives and Pesticide Residues, December, 1969 ; and the Advisory Panel on the Mutagenicity of Pesticides, the Secretary's Commission on Pesticides and their Relationship to Environmental Health, December, 1969. Such recommendations not only reflect critical concerns as to the public health implications of chemical mutagenesis, but also the recent development of a variety of test methods, data from some of which have a high degree of presumptive human relevance.

Chemical Mutagenesis in Mammals and Man is the first of several recent books addressed to this problem with particular emphasis on the importance of in vivo mammalian systems in testing for chemical mutagens. This book is a compilation of papers presented at a symposium held on October 7, 1969, in Mainz, Germany. The editors, Drs Vogel and Röhrborn, are admirably qualified for this purpose, as they are internationally recognized and respected workers in the respective fields of population genetics and mutagenicity testing in mammals.

The book contains thirty chapters by authors who include many well known authorities in chemical mutagenesis, including Barthelmess, Ehling, Kuhlmann, Legator, Malling, and Chu. Most chapters deal comprehensively with the specifics of mutagenicity testing in mammalian systems. The editors have also contributed chapters on genetic monitoring of human populations and on the dominant lethal assay in rodents, and also a very useful summary. The stylistic heterogeneity of the book, which the editors have wisely preserved, makes for dynamic and stimulating reading.

The editors are to be warmly congratulated for their timeliness in publishing this volume, which will be of great value, not only to research workers in the field, but also to the chemical and pharmaceutical industries, and to government agencies.

SAMUEL S. EPSTEIN

\section{Designing Libraries}

New Library Design: Guide Lines to Planning Academic Library Buildings. By Stephen Langmead and Margaret Beckman. Pp. ix +117. (Wiley: Toronto and Chichester, August 1971.) £5.75.

THE ideas for this book came from the authors' collaboration as project architect and systems librarian for the McLaughlin new library building at the University of Guelph, Canada. They have subsequently acted as consultants to several other Canadian libraries and they lecture in library architecture at the Graduate School of Library and Information Science at the University of Western Ontario. Experience taught them that there are certain steps fundamental to the planning process which are ignored at peril and others which, while not absolutely essential, greatly smooth the passage of a plan through its various stages. In consequence they have set down a systematic schedule of steps to be taken from beginning to end of the planning of a large university library. The resulting guidelines enable anyone planning a new academic library to identify the problems which will need to be solved, make it quite clear who will need to be consulted and why, and provide many of the factual details and most up-to-date accepted standards which both architect and librarian will need. As an illustrative example the McLaughlin Library and its planning brief are described, with added diagrams and photographs.

This must surely be a classic case of joint consultation, the very antithesis of the Columbia Law Library which was described as "reflecting, by its symmetry and balance, the triumph of the Architect over the Librarian". Here the librarian takes his proper place as a 